\title{
Computing power
}

\author{
Theoretical approaches have an important role to play in driving forward new ideas in \\ nanoscience and technology, as illustrated by two papers in this issue.
}

Advances in computational techniques mean that it is now possible to predict the results of some experiments without actually having to run them. Supercomputers that can perform tens of trillions of operations per second are the theorist's equivalent of a 24-hour chemistry lab, capable of exploring the properties of numerous combinations in the pursuit of new materials and structures.

In fact, a supercomputer may be one of the most impressive new hires at a new nanocentre. High-performance clusters, such as that recently installed at the Centre for Nanoscale Materials at the Argonne National Laboratory, combined with cleverly designed algorithms, are now fast enough to calculate - from first principles - the properties of nanomaterials containing between five and fifty atoms. According to Michael Sternberg, a scientist at Argonne, this level of computing power is already making an impact in areas such as catalysis, where the ability to computationally screen the properties of hundreds of different metal nanoclusters will allow researchers to improve the energy efficiency of industrial processes and fuel cells.

But what place is there for purely computational studies - rather than those tailored to specific experiments - in a field as applied as nanotechnology? In the last year, Nature Nanotechnology has published only a handful of such papers ${ }^{1-3}$. One reason for this may be that increasing the number of impurity atoms in a nanoscale device from one to two can completely change the way it operates. The need to deal with this level of detail makes it difficult to determine the more general properties of nanosystems.

Addressing such complexity involves pushing the limits of well-established computational techniques that, at present, can only handle small numbers of atoms and molecules, or that rely on simplifying assumptions to treat bulk crystalline solids. Moreover, devices must be considered in the real environment in which they will be measured, which often requires merging calculations that are designed for quantum systems (such as single molecules) with those geared toward macroscopic lengthand timescales (such as a fluid bath or measurement probe).

The best theory papers will also stimulate researchers to try new measurements or revisit old experimental data in a significantly different light. For this to happen, theorists must show an awareness of the types of structures and devices that can - either now, or in the near future - be reasonably engineered, manipulated and measured, and an appreciation of how they will be affected by their environment. At the same time, such predictions must also be relevant to more than one system and set of conditions.

Consider, for example, the use of density functional theory (DFT) to calculate the electronic properties of materials from first principles. A problem that had puzzled materials scientists for some time was the apparent loss of ferroelectricity in ultrathin films, such as those used in capacitors and field-effect transistors. However, it is only recently that DFT has been developed to the stage where it can treat the interface between two insulators ${ }^{4}$ or between an insulator and a metal ${ }^{5}$ in the presence of an electric field, which is essential for understanding the ferroelectric materials used as insulators in actual devices. DFT calculations have shown that the problem depends not only on the ferroelectric film, but also on the ferroelectric-metal inteface ${ }^{6}-$ a result that was subsequently confirmed in experiments with X-rays ${ }^{7}$.

On page 46, Nicola Spaldin and co-workers extend these computational techniques to treat the interface between an insulator and a magnetic metal, and go on to propose a new device called a 'spin capacitor' that can store electron spin in the same way that traditional capacitors store electric charge ${ }^{8}$. According to Spaldin, DFT calculations are able to provide valuable information because the critical length scale for many devices is only a few unit cells and the computations fit - just - in the computer. At the same time, various deposition techniques allow experimentalists to build up structures atom by atom and tailor the device exactly to the specification of theorists ${ }^{9}$.

On page 22, meanwhile, Erio Tosatti and colleagues predict, on the basis of DFT calculations, that platinum nanowires should display an unusual effect called colossal magnetic anisotropy ${ }^{10}$. This proposal is timely because experimentalists are increasingly able to measure the effects of magnetization on single-electron transport in such nanowires.

As computational approaches continue to catch up with advances in experimental techniques, we look forward to publishing more theoretical papers that push nanoscience and technology as far as it can go.

\section{References}

1. del Valle, M., Gutierrez, R., Tejedor, C. \& Cuniberti, G. Nature Nanotech. 2, 176-179 (2007).

2. Lehmann, J., Gaita-Ario, A., Coronado, E. \& Loss, D. Nature Nanotech. 2, 312-317 (2007).

3. Gong, X. et al. Nature Nanotech. 2, 709-712 (2007).

4. Souza, I., fñiguez, J. \& Vanderbilt, D. Phys. Rev. Lett. 89, 117602 (2002).

5. Stengel, M. \& Spaldin, N. A. Nature 443, 679-682 (2006).

6. Junquera, J. \& Ghosez, P. Nature 422, 506-509 (2003).

7. Fong, D. D. et al. Science 304, 1650-1653 (2004).

8. Rondinelli, J. M., Stengel, M., \& Spaldin, N. A. Nature Nanotech. 3, 46-50 (2008).

9. Ramamoorthy, R. Nature Nanotech. 3, 7-8 (2008).

10. Smogunov, A., Dal Corso, A., Delin, A., Weht, R. \& Tosatti, E. Nature Nanotech. 3, 22-25 (2008). 